\title{
Erratum to: Microfossil assemblages and relative sea-level fluctuations in a lagoon at the Oxfordian/Kimmeridgian boundary (Upper Jurassic) in the eastern part of the Paris Basin
}

\author{
Apolline Lefort • Bernard Lathuilière • \\ Cédric Carpentier • Vincent Huault
}

Published online: 25 February 2011

(c) Springer-Verlag 2011

\section{Erratum to: Facies}

\section{DOI 10.1007/s10347-010-0259-4}

Unfortunately, the legend of the Fig. 6 was incorrect.

The correct version is as follow:

Fig. 6 Microfossils of the Upper Oxfordian-Lower Kimmeridgian from the Gudmont-Villiers section. a Labyrinthina mirabilis Weinschenk and Labalina (Calcaires crayeux de Gudmont Formation). b Nautiloculina oolithica Mohler (CCG Formation). c Mohlerina basiliensis (Mohler) (Calcaires à serpules de Gudmont Formation). d Hungarillina pedunculata Blau \& Wernli (Calcaires à serpules de Gudmont Formation). e Troglotella incrustans Wernli \& Fuchs in Cladocoropsis mirabilis Felix (Calcaires crayeux de Gudmont Formation). f Pink Lenticulina (Calcaires à astartes Formation). g, i Everticyclammina virguliana (Koechlin), h Alveosepta jaccardi (Schrodt) (CCG Formation) (Oolithe de Lamothe Formation). j Dasycladacean (Salpingoporella pygmae Guembel) G6. k Cayeuxia piae Frollo (G'9). I Lithocodium aggregatum Elliott (Calcaires crayeux de Gudmont Formation). When the scale is not precised, the segment measures $500 \mu \mathrm{m}$

The online version of the original article can be found under doi:10.1007/s10347-010-0259-4.

A. Lefort $(\bowtie) \cdot$ B. Lathuilière · C. Carpentier · V. Huault Laboratoire G2R, Nancy-Université,

54506 Vandoeuvre-lès-Nancy Cedex, France

e-mail: apolline.lefort@g2r.uhp-nancy.fr 